\title{
Políticas de población y salud reproductiva en el Paraguay
}

\author{
Population policies and reproductive health \\ in Paraguay
}

Luis Carlos Simancas 1

Mónica Ruoti de García de Zúñiga 2

\footnotetext{
${ }^{1}$ Instituto Paraguayo de Estudios Sociales. Juan de Salazar 830 Asunción, Paraguay. 2 Departamento de Ciencias Sociales, Instituto de Investigaciones en Ciencias de la Salud, Universidad Nacional de Asunción. IICS-UNA.

Río de la Plata y Lagerenza. Casilla de Correo 2511, Asunción, Paraguay.
}

\begin{abstract}
The population's high growth rate, age profile, and geographical distribution have aroused increasing public concern in Paraguay. The country is involved in a moderate demographic transition, compatible with the consequences of modernity and the uneven rate of both social and economic changes. Reduction of mortality and the persistence of high birth rate patterns result in an age structure that consolidates demographic growth, with an increased focus on the dependent population. In the late 1960s the need for a systematic approach to population problems was perceived within the framework of economic planning. National governments had, and currently have, an ambivalent perception of this issue, since population growth is simply considered a positive factor. This concept results from an economic view of the consequences of a reduced domestic market in absolute terms. The lack of a Development Plan, the management deficit, and the shortage of training leave doubts as to the establishment of organically connected policies or programs concerning population.
\end{abstract}

Key words Reproductive Health; Demographic Policies; National Health Programs; Family Planning Policy; Demograph

Resumen El acelerado crecimiento de la población, su conformación por edades y su distribución espacial han despertado en el Paraguay una creciente preocupación pública. Experimentamos una transición demográfica moderada, conformada por las consecuencias de la modernización y el ritmo dispar de los cambios económicos y sociales. Al disminuir la mortalidad y persistir patrones de alta natalidad, se conforma una estructura de edades que consolida el crecimiento demográfico, con un aumento centrado en la población dependiente. A fines de los sesenta se percibió la necesidad de abordar de forma sistemática la problemática de población, principalmente en el marco de la planificación económica, implantando al mismo tiempo la planificación familiar como instrumento de salud pública, antecedente de lo que es hoy salud reproductiva. La percepción de los gobiernos nacionales sobre el tema de población fue y es ambivalente, porque se considera el crecimiento poblacional como un factor siempre positivo, producto de una visión economicista que absolutiza las consecuencias de un mercado interno reducido. La inexistencia de un plan de desarrollo, el déficit de gestión y la insuficiente capacitación tornan dudosa la posibilidad de definir políticas o programas de población orgánicamente articulados, excepto en el ámbito de la salud reproductiva.

Palabras clave Salud Reproductiva; Política Demográfica; Programas Nacionales de Salud; Política de Planeamiento Familiar; Demografía 


\section{Evolución demográfica}

En las últimas décadas, el acelerado crecimiento de la población, su conformación por edades y su distribución espacial han despertado en el Paraguay una gradual pero sostenida preocupación pública. Se configuraron problemas cuya comprensión supone el esclarecimiento de las interrelaciones entre población, desarrollo y medio ambiente, insertas en un modelo político-social viable. Sin embargo, persisten las divergencias sobre el sentido y las implicancias de tales fenómenos, y principalmente sobre lo que debería hacerse, pese a las conferencias mundiales del Cairo y Beijing, cuyos planes de acción han sido aprobados - con ciertas resalvas - por el gobierno nacional.

\section{Tamaño y evolución de la población}

En el Paraguay se realizaron una serie de censos de población de muy disímil valor, uno de los primeros, relevado y publicado en 1899/ 1900, totalizó 696.392 habitantes, luego se registraron 650.541 personas en 1914, ascendiendo posteriormente a 828.968 en 1926 en tanto que, en 1936, los residentes totalizaron 992.420 habitantes. Las tasas intercensales en dicho período fueron de (-)0,48; 2,04 y 1,82, respectivamente, con un crecimiento absoluto en el ciclo de 296.028 habitantes. La tasa promedio del período $1900 / 1936$ es de 0,99 , aunque tales censos adolecían de severas restricciones metodológicas, hecho que los torna en referencias históricas que deben usarse con extrema prudencia.

En 1950 la población englobaba a 1.328.452 habitantes, con una tasa de crecimiento intercensal (1936-1950) de 2,18, acrecentándose en 1962 a 1.819.103, con una tasa de 2,7 y un incremento absoluto de 534.968 personas $(40,26 \%)$. En el decenio siguiente (1962-1972), la población creció a una tasa ligeramente inferior, de 2,5 , totalizando 2.357 .955 habitantes, con una diferencia absoluta con el censo anterior de 538.858 , lo que representa una tasa de crecimiento natural de 29,62 por ciento.

Durante décadas el crecimiento de la población se mantuvo relativamente estable, y la composición por edades de la población paraguaya persistió en una cierta estabilidad, con preponderancia de menores de 15 años del orden del $44 \%$ en 1950 , del $41 \%$ en 1982 y del 41,5\% en 1992 (DGECE, 1950; 1962; 1972; 1982; 1992).

Respecto de la estructura por sexos, sólo se registran modificaciones de cierta relevancia considerando el lugar de residencia (urbano o rural). El Paraguay ha pasado de ser un país eminentemente rural, - con un $65 \%$ de población campesina, según el censo de 1950 -, a congregar el 50,5\% de la población en zonas urbanas, de acuerdo al censo de 1992, y la tendencia se presenta como irreversible.

Existe razonable consenso en que nos encontramos en el principio de una transición demográfica moderada, sobredeterminada por las consecuencias previsibles y combinadas, de una incipiente modernización y la asincronía en el ritmo de los cambios económicos y sociales. Al disminuir la mortalidad y persistir patrones de alta natalidad, se conforma una estructura de edades que tiende a consolidar el crecimiento demográfico, con un aumento centrado precisamente en la población dependiente. Así, y de mantenerse la asimetría e inequidad de la distribución de la riqueza, las características del crecimiento poblacional incidirán negativamente en los intentos por mejorar la calidad de vida de los sectores pobres. De modo adicional, generan el descreimiento en las políticas económico-sociales, que no enfrentan de modo directo los severos condicionamientos imputados a las altas tasas de natalidad.

\section{Fecundidad y mortalidad}

La Tasa Global de Fecundidad (TGF) ha descendido de 6,8, en 1950-55, a 4,9 al inicio de la década del 70, y se mantiene prácticamente estable hasta $1990(4,7)$, con diferencias substanciales entre la población urbana $(3,6)$ y rural $(6,1)$ (Cepep/DHS, 1990). En 1995 se observa una pequeña disminución, ya que la TGF se redujo a 4,5, manteniendose las diferencias de fecundidad entre la población urbana $(3,3)$ y la rural (5,8) (Cepep/CDC/Usaid, 1995/1996).

La fecundidad máxima recae en el grupo de mujeres de 25 a 29 años, por lo que se la tipifica como de fecundidad de cúspide tardía. La tasa de fecundidad general (TFG) por mil mujeres de 15 a 44 años es de 159 y la tasa bruta de natalidad es de 33 nacimientos por mil personas (Cepep/DHS, 1990), valores que poco difieren desde la Encuesta Nacional de Fecundidad de 1979 (DGEC, 1981) ( TGF = 152 y $\mathrm{TBN}=35$ ). Las mujeres en edad fértil representan el 24,2 por ciento de la población total, proporción que es considerada alta.

La dinámica demográfica del decenio de los 90 está singularizada por un rápido crecimiento poblacional $(3,1)$, derivado de la persistencia de las tasas de fecundidad $(4,7)$, al descenso de la Tasa de Mortalidad Infantil (46,7 por mil) y a una cierta estabilización del saldo migratorio positivo (1 por mil). Se considera 
que el país se halla en el inicio de un proceso de transición demográfica caracterizado por altas tasa de fecundidad que coexisten con un descenso de la tasa de mortalidad.

La primera relación sexual ocurre, en promedio, dos años antes de la primera unión, aunque se registran diferencias importantes en el intervalo transcurrido entre la primera relación sexual y el nacimiento del primer hijo. Considerando la edad mediana, las jóvenes de zonas urbanas tienen su primera relación a los 19,3 años y el primer hijo a los 22,4 años, en tanto que las de áreas rurales tienen la primera relación a los 19,5 años y el primer hijo los 20,3 años (Cepep/DHS, 1990).

La Tasa Bruta de Mortalidad descendió de 11,09 a 6,68 por mil, en el período de 1960 1990, lo que refleja una reducción cercana al 40 por ciento, explicable básicamente por la disminución de la mortalidad infantil (TMI), que varió de 92,7 por mil (MSPBS/OPS, 1989) a 35,4 en el período considerado (Cepep/DHS, 1990).

La disminución de la TMI se refleja diferencialmente en las áreas rurales $(16,5 \%)$ y en la capital (32\%) o en ciudades mayores del interior del país (25,6\%) (Cepep/DHS, 1990; DGECENF 79, 1981). Correlativamente aumenta la esperanza de vida, que pasa de 62,2 años, en 1950-55, a 67,59 en 1985-90.

En 1960 la tasa de mortalidad materna (TMM) era de 2,7 por mil nacidos vivos, inferior a las registradas en los años 1968 y 1976, que fueron de 6,0 y 6,3, respectivamente (MSPBS/ OPS, 89:43). Este comportamiento errático sólo es explicable por deficiencias en el registro. Persiste una alta tasa de mortalidad materna (1,7 por mil nacidos vivos) relacionada a complicaciones obstétricas (hemorragias, sepsis y abortos) o a derivaciones de procedimientos médicos practicados durante el parto.

\section{Políticas de población}

\section{Antecedentes}

La preocupación por los temas de población es recurrente en el desarrollo del Paraguay, dado que su inserción en la historia occidental fue mediada por los procesos de poblamiento en la época colonial (Velazquez, 1972), y su persistencia como sociedad autónoma estuvo condicionada por dos guerras, que fungen como sobredeterminantes negativas, particularmente en lo demográfico.

No obstante, recién a fines del decenio de 1960 - a tono con lo sucedido en la región -, se percibió la necesidad de abordar de un modo sistemático la problemática de población, en el marco de la planificación económica. Aun cuando se partiera de concepciones opuestas, estructuradas en centros académicos externos y eventualmente propensas a la descalificación mutua, se intentó esclarecer vinculaciones entre variables socioeconómicas y las relativas a la población, para determinar cuál es el papel del Estado en las interrelaciones entre economía y sociedad.

A nivel internacional, los estudios demográficos adquieren relevancia en el ámbito de las Ciencias Sociales, coincidiendo con la decisión de varios gobiernos regionales de definir el rol del Estado en la planificación económico-social, con inclusión de medidas que, de modo dispar, intentaron incidir en el crecimiento y distribución de la población. La reflexión sobre la posibilidad de esclarecer las interrelaciones entre población y desarrollo y de orientar su configuración en un sentido determinado tuvo como trasfondo las tendencias previsibles de fecundidad, las tasas de mortalidad y los fenómenos migracionales, que convergían en altas tasas de incremento poblacional.

En el marco regional, la interpretación de las implicancias del crecimiento de la población sufrió variaciones que oscilaron entre el entusiasmo, por las potenciales ventajas atribuidas al reforzamiento del mercado interno, a la expansión de la fuerza de trabajo y el aumento relativo del poder nacional en el contexto de la región; y las visiones que consideraban la acentuación de tasas elevadas de crecimiento poblacional como el factor crítico que impedía - o cuando menos retardaba - el proceso de desarrollo de las sociedades nacionales. De este modo, la poblemática poblacional empezó a girar en torno a los aspectos positivos y negativos atribuidos a tasas altas de crecimiento, y a las medidas para disminuir dichas tasas controlando la fecundidad.

La perspectiva académica sugiere que las políticas de población deben ser diseñadas dentro de un marco conceptual que involucre a la mayor cantidad de factores pertinentes. En este sentido, el análisis de las interrelaciones y los nexos causales entre crecimiento poblacional y desarrollo económico-social no se hallan suficientemente develados y establecidos, como para aceptar con fuerza conclusiva que el crecimiento de la población es la variable más importante a controlar para consolidar el desarrollo. Además, los sistemas de planificación sólo recientemente incorporaron a la población y a los elementos ambientales como variables constitutivas del proceso de planificación. 
En el contexto teórico existente a nivel global, coexistieron básicamente dos tipos de formulaciones de política (ONU, 1974):

- una de carácter extremadamente general, que, por abarcante, perdía capacidad operativa, ya que no podía diferenciarse de las medidas políticas relativas a campos específicos, como la economía, la salud o la educación, aunque se le atribuye la virtud de obligar a los planificadores a evaluar las consecuencias demográficas de las decisiones;

- otra de índole restrictiva, centrada casi exclusivamente en la planificación familiar, legitimada por el hecho de tornar accesible la posibilidad de una paternidad responsable y preservar las condiciones de salud materna e infantil, y también como medio de influir en la fecundidad.

En cierto modo, la mayoría de las aproximaciones a políticas de población pagaron tributo a una o ambas posiciones. Las concepciones implícitas entre estas posturas extremas evolucionaron de modo dispar en el ámbito regional, hasta constituir, entre otras posibles, dos posiciones relevantes y bien diferenciadas.

La primera centra sus esfuerzos en la compatibilización permanente entre dinámica demográfica y estilos de desarrollo, con particular acentuación en el crecimiento de la población. Así, la presión demográfica sobre la estructura de servicios es identificada específicamente como factor restrictivo del proceso de desarrollo, y las consecuencias negativas atribuidas se extienden desde la falta de escuelas hasta la polución ambiental. Tal perspectiva centra la solución de los problemas en el control de la fecundidad, no como expresión volitiva de autonomía personal o familiar, sino como medida con pertinencia técnico-administrativa, generada desde la racionalidad de la planificación económico-social, en función de determinadas metas del desarrollo.

Una concepción tal es proclive a considerar como natural la confrontación entre la autonomía del comportamiento reproductivo de las personas o de las parejas, y la tasa de crecimiento aceptable para la sociedad en su conjunto, definida por técnicos en planificación.

Por otra parte, considerando la influencia de lo demográfico en lo económico, persiste la arcaica necesidad de preguntarse si somos pobres porque crecemos mucho o son los pobres que crecen mucho por el hecho de serlo. Hay que dilucidar si el crecimiento demográfico es efectivamente la causa de nuestra pobreza o si es la pobreza la causa de un crecimiento desmedido de la población pobre. Polarizando e invirtiendo los términos del problema, parece más racional relacionar la pobreza con la asimetría en la distribución de la riqueza y con la forma de apropiación del excedente económico, antes que hacerlo exclusivamente con las tasas de crecimiento poblacional. Sin embargo, tampoco es razonable esperar a que existan situaciones óptimas de justicia, equidad y solidaridad social irrestricta - en ámbitos nacionales e internacionales - para comenzar a considerar los efectos demográficos en el sistema económico. Por tanto, sin perder la perspectiva de los principios o de lo que se asume como puntos de partida legítimos, hay que considerar la viabilidad real - social - de las propuestas de política, y seleccionar acciones interventivas-correctivas que sean realizables dentro de los condicionamientos objetivos existentes, incluyendo las medidas demográficas.

La segunda posición se estructuró en torno a la vigencia real de los derechos individuales, para lo cual resulta imprescindible la reducción de las desigualdades sociales, nacionales e internacionales. Las diferencias en las tasas de mortalidad materna e infantil en desmedro de los sectores pobres, en gran medida, son consecuencia de estilos de vida inadecuados y de la asimetría en el acceso - cultural y económico - a los servicios, atribuibles a las disparidades en ingreso, educación y condiciones básicas de vida. Se aduce que es posible construir una práctica consensuada, supuesto que el comportamiento reproductivo se asuma mediando insumos adecuados para la toma de decisiones.

\section{Percepciones gubernamentales sobre cuestiones de población}

Históricamente la percepción de los gobiernos nacionales sobre las implicancias de la problemática poblacional fue y es ambivalente, dado que se insiste en considerar al crecimiento poblacional como un factor permanentemente positivo, inducido - probablemente - por una visión economicista que tiende a absolutizar las consecuencias de un mercado interno reducido, sin considerar suficientemente otros factores que intervienen en el proceso de desarrollo. El argumento de economía de escala o de mercado reducido ha perdido validez por la vigencia del Mercado Común del Sur (Mercosur).

La posición gubernamental ha sido, tradicionalmente, de no intervención en la tasa de crecimiento poblacional; sin embargo ha promovido programas de salud materno-infantil, con componentes de planificación familiar de efectividad variable, además de la creación de 
pequeños programas de empleo de diseño puntual, restringidos adicionalmente por la inexistencia de políticas sociales donde insertarse orgánicamente. La reforma agraria y la colonización constituyeron los programas oficiales que, directa y pertinentemente, son vinculables a los procesos de redistribución de la población.

Documentos de la Secretaría Técnica de Planificación (STP, 1976; 1984), referidos a la dinámica de población, resaltaban explícitamente la necesidad de aumentarla, aunque advertían sobre los requerimientos de servicios sociales que el sistema económico debía solventar. Existió una posición pro-natalista declarativa, no siempre reflejada en los planes sectoriales de salud y educación, que tendía a facilitar información y a proveer servicios orientados a conformar volitivamente el tamaño de la familia, en el marco de una paternidad responsable y servicios de promoción de conductas basadas en el autocuidado.

El Plan Nacional de Desarrollo (1985-1989) afirma (STP, 1984) la interrelación existente entre situación social y aspectos económicos, sociológicos y políticos. Señala como desafío básico del crecimiento con redistribución del ingreso la superación de restricciones estructurales y de coyuntura, para lograr que los beneficios del desarrollo fluyan con equidad a los destinatarios finales. Estima que la cuestión social no puede constituirse en un elemento marginal en las directrices generales de asignación de recursos, que debe ser asumida como una inversión que funciona simultáneamente como causa y efecto de los procesos de desarrollo social, y no como un gasto siempre evitable ante la permanente escasez de recursos.

\section{Evolución de la política de población en el país}

La intervención del Estado es una constante en la historia nacional, y en lo que respecta a temas de población, su acción se vincula con la problemática de salud y bienestar familiar, con la reforma agraria y la creación de polos de desarrollo, antes que a consideraciones de naturaleza conceptual referidas a la elaboración de un modelo de sociedad y el delineamiento de estrategias de desarrollo, proceso de planificación en el cual la dinámica poblacional tiene un papel insoslayable.

Los diversos gobiernos nacionales evitaron de manera sistemática la formulación de políticas explícitas de población, especialmente las demográficas, eludiendo formalmente programas tendientes a la modificación de las tasas de natalidad. Al parecer se ha reconocido la conveniencia de no establecer metas demográficas y simultáneamente proveer servicios especializados que reclaman sectores de la comunidad. La neutralidad de esa posición ha sido, en determinados períodos, sólo aparente ya que los compromisos asumidos en la prestación de servicios fueron de magnitud y características tales, que es posible asignarle efectos demográficos aunque de muy difícil cuantificación.

\section{Lo que dice la constitución}

La Carta Magna del Paraguay consagra, en su Artículo 4, el derecho a la vida, y garantiza su protección, en general, desde la concepción. Obviamente, "en particular" se puede legislar y aceptar el aborto, tal como está sucediendo ahora en el tratamiento del nuevo código penal.

El Artículo 6 establece la necesidad de realizar estudios sobre la realidad poblacional del Paraguay, en tanto que, en el 61, se reconoce “... el derecho de las personas a decidir libre y responsablemente el número y la frecuencia del nacimiento de sus hijos, así como a recibir, en coordinación con los organismos pertinentes, educación, orientación científica y servicios adecuados a la materia. Se establecerán planes especiales de salud reproductiva, y salud materno infantil para la población de escasos recursos". Dado que este derecho se refiere a las "personas", puede interpretarse que incluye a hombres y mujeres adultos, así como a jóvenes que todavía no hayan constituido familia.

\section{Estrategias}

En diciembre de 1991 una Misión de Revisión de Programa y Desarrollo de Estrategias del Fondo de Población de las Naciones Unidas (FNUAP), con la colaboración - o cuando menos con la aprobación - del Gobierno, identificó y definió la estrategia (FNUAP, 1991) (Onu/Cepal/Celade, 1993) que orientaría las intervenciones venideras en el área de población.

Los programas del sector público por lo general se han atenido formalmente a los delineamientos generales recomendados por el FNUAP. En tanto que las organizaciones privadas han desarrollado estrategias y acciones interventivas inducidas por entidades patrocinantes del exterior, especialmente en el campo de la planificación familiar, ofreciendo incluso servicios de esterilización masculina y femeni- 
na. Y dado que no existe un Plan Nacional de Desarrollo que especifique políticas o programas de población, se torna muy difícil evaluar lo que sea esa referida "estrategia de población”.

\section{Estrategias globales}

El documento define como "estrategias globales" la:

1) toma de conciencia sobre población y desarrollo: apela a que los niveles de decisión y técnicos esclarezcan la relación entre población y desarrollo; y dado que la temática poblacional no constituye prioridad se torna imprescindible impulsar debates e investigaciones, apoyados por acciones de difusión, que finalmente generen una masa crítica eficazmente sensibilizada ante lo poblacional;

2) participación comunitaria: se menciona la falta de participación comunitaria como un déficit que impide impulsar y ordenar el desarrollo social; por tanto, se recomienda la promoción de todas las formas de participación comunitaria, empleando la capacitación, la asesoría y la captación de líderes comunitarios como instrumentos privilegiados de acción;

3) descentralización y administración municipal: la implementación de planes y programas no escapa a la constante de un Estado históricamente centralizado, que interfiere y obstaculiza un buen desempeño. Existe una creciente aceptación de las ventajas de la descentralización, pero se está lejos de una visión razonablemente compartida de lo que conceptualmente ésta sea y más distante todavía de un consenso operativo. Se recomienda realizar esfuerzos en concretar la descentralización, confiriendo mayor autonomía a los municipios y apoyo a entidades locales en el diseño y ejecución de programas de planificación familiar. Para ello se priorizan dos aspectos: capacitación administrativa y un plan de comunicación social;

4) cooperación sector público-privado: se reconoce la disposición del Gobierno a cooperar con el sector privado de las ONG's y canalizar su experiencia en los programas existentes, principalmente en los ámbitos de salud y educación. Se recomienda, por tanto, diseñar e implementar acciones que complementen las potencialidades de los sectores público y privado en programas de población, con inclusión de estudios e investigaciones sobre el tema;

5) coordinación intersectorial e interagencial: la falta de coordinación entre sectores y agencias, traducida en una suerte de segmentación inarticulada del espacio institucional, es una de las falencias que más negativamente inciden en la falta de efectividad de la asistencia técnica. Se recomienda el reforzamiento de las estructuras de coordinación interinstitucional para finalmente llegar a una instancia nacional de regulación;

6) formulación de una política de población: existe consenso generalizado que la definición de políticas explícitas contribuiría cuando menos a establecer un marco para orientar y armonizar las acciones del sector público y del privado.

\section{Estrategias sectoriales}

En el apartado de "estrategias sectoriales" se mencionan las siguientes:

1) población y desarrollo: ante la inexistencia de un plan global de desarrollo, las recomendaciones deben referirse obligadamente a la planificación sectorial, especialmente en las áreas de salud y educación. La coordinación de estas acciones sectoriales es prerequisito de efectividad. Se recomienda, por tanto, capacitar en dinámica de población a funcionarios responsables de planificar y ejecutar dichos programas; reforzar los niveles de coordinación intersectorial; diseñar investigaciones que esclarezcan las interrelaciones entre variables demográficas y socioeconómicas; consolidar la preservación de los recursos naturales y promover la defensa del medio ambiente; incorporar el tema de población y medio ambiente en las campañas de sensibilización y educación;

2) recopilación y análisis de datos en materia de población: la falta de información válida es crónica. Los censos de población sufren altos niveles de omisión y su divulgación es tardía, el subregistro de los datos del Registro Civil es considerable, sobre todo el referido a inscripciones oportunas. En el marco de la descentralización, se recomienda desagregar la información censal a nivel de manzanas en zonas urbanas y de comunidades (compañías) en zonas rurales, para simplificar la toma de decisiones. Se propone la implantación de procedimientos de integración de datos y la capacitación de funcionarios destinados a la certificación y registro de datos vitales.

3) salud materno infantil y planificación familiar: es baja la cobertura de los servicios de planificación familiar con enfoque de riesgo, insertos en programas de salud materno infantil. Dada la magnitud de la demanda no satisfecha, la Misión recomienda emplear un enfoque multisectorial y utilizar canales alternativos de información y comunicación que promuevan 
la demanda y articulen la oferta de servicios. Asimismo, aconseja la realización de un estudio de necesidades como paso previo a la planificación de la oferta de servicios y de anticonceptivos, investigando al mismo tiempo los elementos socio-culturales que inciden en el comportamiento reproductivo. La mujeres campesinas son escogidas como sector prioritario, especialmente las adolescentes;

4) información, educación y comunicación en población: los aspectos resaltantes a ser enfocados son: escaso e inadecuado conocimiento del tema a nivel de funcionarios, alta tasa de embarazo en adolescentes, concientización del público sobre las implicancias del tema poblacional y la insuficiente comprensión de la relación población-desarrollo. El abordaje debe identificar a los diversos grupos sociales y reconocer las dificultades adicionales derivadas del bilinguismo como factores concluyentes en el diseño y ejecución de políticas diferenciadas. El Ministerio de Educación debe proseguir con programas en ciencias naturales, sociales y educación sanitaria, creando una marco referencial que posibilite conexiones integradas. Se recomiendan tres líneas de acción: concientizar a los diferentes actores sociales sobre el tema de población; promover una toma de decisiones informada sobre el comportamiento reproductivo, y mejorar los conocimientos de los dirigentes sobre el tema de población;

5) mujer, población y desarrollo: los programas dirigidos a la mujer han adolecido inicialmente de una suerte de asistencialismo reivindicatorio, cuyo efecto inmediato fue velar la problemática de la mujer como elemento específico y singular de las políticas de desarrollo e impedir su tratamiento desde la perspectiva de género. La estrategia recomendada tiene tres vías de acción: coordinación inter-institucional entre los distintos sectores con ingerencia en el campo de la planificación familiar, educación y salud de la mujer; capacitación del personal de campo, y fortalecimiento de los grupos conformados en el interior de programas sociales ya existentes.

\section{Planes nacionales de salud con incidencia en población}

Los gobiernos nacionales diseñaron y ejecutaron diversos planes de salud.

\section{Acciones sobre la mortalidad (1975/1982)}

Respecto a la mortalidad - obviamente la única política admisible es su reducción - el Ministe- rio de Salud Pública y Bienestar Social elaboró y ejecutó un plan con los objetivos siguientes:

- extensión de los servicios a personas;

- aumento de acciones interventivas sobre el medio ambiente;

- expansión y mejoramiento de los servicios sanitarios.

La directriz fundamental se centraba en la reducción de los riesgos de enfermedad y muerte, atribuibles a insuficiencias en la atención materno-infantil y de las enfermedades transmisibles. Se puso énfasis en la atención rural, el saneamiento ambiental y la provisión de servicios sanitarios básicos.

Posteriormente, el Plan Quinquenal tuvo continuidad en el Plan de Salud para el Bienio 1981/1982, derivado de una evaluación de impacto que propició ajustes operativos.

Subsiguientemente entró en vigor el Proyecto para la Extensión de la Cobertura de los Servicios de Salud, con el objetivo básico de extender la cobertura a toda la población, centrado en asentamientos menores de 2.000 habitantes, y presidido por un sistema de captación y derivación que abarcaba desde la atención primaria hasta casos de alta complejidad. Dicho proyecto, inspirado en la filosofía de "salud para todos en el año 2.000”, tenía metas de aumentar a 70 años la esperanza de vida al nacer; reducir la mortalidad infantil a 30 por mil nacidos vivos; inmunizar al $100 \%$ de los niños menores de 1 año y al $100 \%$ de las embarazadas contra tétano neonatorum; aumentar la cobertura de agua potable y saneamiento básico.

\section{Programas de población y salud familiar}

En relación con la fecundidad, los sucesivos gobiernos se abstuvieron de formular políticas explícitas.

En el decenio de 1960, planificadores y expertos en salud pública detectaron notorias deficiencias en el ámbito de la salud maternoinfantil, coincidiendo con una incipiente demanda urbana de servicios de planificación familiar. Estas actividades específicas se inician en agrupaciones privadas y, entre 1972 y 1978 , son incorporadas oficialmente al Ministerio de Salud Pública y Bienestar Social. Durante el período 1980/1987, los servicios oficiales ofrecieron planificación familiar exclusivamente en base al método Billing, reestableciéndose en 1988 la oferta médica completa en planificación familiar, como parte de un sub-programa de protección familiar en el marco del Programa Nacional de Salud Materno-Infantil. Además, el proceso de modernización que afectó principalmente a las áreas urbanas con la con- 
secuente expansión de la escolarización, el ingreso de la mujer al mercado de trabajo y la urbanización contribuyeron espontáneamente a incrementar la demanda por servicios de planificación familiar para adecuar el tamaño familiar a nuevas concepciones culturales.

\section{Programas de educación en población}

El Ministerio de Educación y Culto (MEC) por medio del Departamento de Curriculum, inserta los temas de población en los planes de estudio desde el nivel primario hasta el nivel superior. Los temas de Educación en Población se adecuan a las especificidades de distintas materias, tales como, Estudios Sociales, Ciencias Naturales y Comunicación, entre otras.

Las actividades relativas a Educación en Población encuentran su antecedente inmediato en el Proyecto PAR/79/P10 con el FNUAP, cuyos objetivos específicos son: enriquecer el curriculum y reforzar el apoyo a los docentes y capacitar a supervisores y técnicos...

Estas acciones tuvieron continuidad en el Proyecto FNUAP/PAR/88/P02 sobre "Educación Familiar y Población”, que realizó un censo entre cuyos objetivos figuraba el de "detectar la validez y el grado de aceptación de los contenidos de Educación Familiar y Población incluidos en el nivel primario".

Plan nacional de salud reproductiva (nu-1996) (MSPBS-FNUAP-OPS, 1996)

Entre los años 1988 y 1992, el Fondo de Población de las Naciones Unidas (FNUAP) conjuntamente con la Organización Panamericana de la Salud (OPS), mediante el Proyecto PAR/88/ P01, decidieron apoyar el Programa Nacional de Salud Familiar, cuyos objetivos eran fortalecer la capacidad del programa integral de salud materno infantil... y contribuir a implementar la planificación familiar, el control prenatal, la atención del parto institucional y del post parto, control del crecimiento y desarrollo del niño, la detección oportuna del cáncer de cuello uterino y de la mama.

En 1993 se diseñó y ejecutó un proyecto puente de "Apoyo a la Salud Reproductiva y Planificación Familiar", con el propósito de conferir continuidad a las acciones emprendidas con anterioridad. Finalmente, en 1996, se elabora el Plan de Salud Reproductiva y Planificación Familiar, cuyo objetivo general es:

"Contribuir al desarrollo de la capacidad nacional y al mejoramiento de los programas, proyectos y servicios de salud reproductiva y favorecer los procesos de desarrollo integral de la familia, de la mujer y del adolescente y de la comunidad; al conocimiento y aplicación de los derechos reproductivos; potenciar la igualdad y la equidad entre hombres y mujeres; y contribuir con los aspectos de salud, educación, legales y laborales que se vinculan con la problemática de la salud reproductiva y la planificación familiar".

El plan abarca áreas de salud, educación, mujer y desarrollo, información-educación-comunicación, participación social e investigación. Adopta como estrategia la conformación de un Consejo Nacional de Salud Reproductiva y Planificación Familiar, integrado por representantes de instituciones públicas nacionales y de cooperación técnica, con la directriz de concentrar y ordenar los esfuerzos. Estratégicamente, se trata de concertar institucionalmente, de movilizar recursos, de investigar y difundir información válida, de informar, educar y comunicar; de organizar la participación social y la comunitaria, de capacitar y desarrollar recursos humanos, simultáneamente con el fortalecimiento de los espacios locales de acción directa y el mejoramiento de los servicios para facilitar su acceso, para todo lo cual se requiere la participación y cooperación técnica y financiera de las instituciones internacionales.

\section{El plan de desarrollo social}

El gobierno nacional, con asesoramiento del Pnud, elaboró un plan de desarrollo social (SAS, 1996), con el proposito de responder a las crecientes demandas enraizadas en el empobrecimiento creciente de la poblacion y en el deseo de consolidar la demoracia como forma de gobierno. Un análisis detallado del plan excede los propositos del trabajo; sin embargo, es pertinente señalar con énfasis que, a pesar de la densidad técnica y de su enfoque participativo, no incorpora la población como eje de concepción. No es de extrañar la existencia de contradicciones en los delineamientos de políticas sujetos a visiones diferentes y recursos permanentemente escasos, pero creer que la población es sólo un dato dado para la definición de políticas sociales es ignorar su papel en los procesos de desarrollo, en los políticos y en la planificación misma. Es generalmente aceptado que las políticas sociales son complejas y, por lo mismo, proclives a guardar bolsones de incoherencias. Un factor que podría reducir este riesgo es que las políticas sociales estén sustentadas o conlleven directrices sobre el tema de población. 


\section{Interrogantes a modo de conclusión}

Uno de los problemas fundamentales que acarrea la complejización de las interrelaciones sociales es la consagración del poder del peritaje y la progresiva disminución o la vanalización del debate público. Los criterios de decisión se convierten en criterios técnicos y se abandonan los criterios de sentido. Esta perspectiva es particularmente importante en lo que se refiere a población, ya que puede darse una suerte de confiscación de la posibilidad de decidir ejercida por el peritaje, grave para el ciudadano quien, en el proceso de mediación efectuado por el saber técnico, pierde la perspectiva del fin último y sólo percibe la realidad de la coacción que permean las decisiones políticas.

Persisten algunos interrogantes sobre la naturaleza de las percepciones en relación a las cuestiones de población, que en definitiva plantean tres abordajes posibles: como problema demográfico; como limitaciones de los factores demográficos al logro de metas de desarrollo; y como vínculos entre los factores de población, medio ambiente y desarrollo.

A la natural complejidad del tema se agrega la incapacidad gubernamental para definir cuál es el núcleo de nuestra problemática poblacional. A tenor de las últimas tendencias, se sugiere que la problemática poblacional paraguaya sea planteada básicamente como problema demográfico, ya que parece constituir un modo adecuado de abordar las implicaciones más visibles de las cuestiones de población. Y si el problema es demográfico, las variables pasibles de intervención son la fecundidad, la migración y la mortalidad. Respecto de la última, social y moralmente sólo es admisible su reducción y si la migración es de difícil control, resta la fecundidad como la única variable para ejecutar políticas de población.

Además, la inexistencia de planes de desarrollo, conjuntamente con falta de información adecuada y fiable, tornan prácticamente inviable la posibilidad de plantear en otros términos dicha problemática.

Pero la adopción de esta perspectiva sobredetermina algunos aspectos del problema que es necesario dilucidar: en qué consiste exactamente nuestro problema demográfico ¿crecimiento, tamaño, estructura, distribución, densidad? Qué tipo de enfoque es pertinente: ¿parcial o contextual, holístico o sectorial? Tampoco hemos definido si tenemos algún ideal de población, y si el hacerlo iimplica establecer metas demográficas cuantitativas?

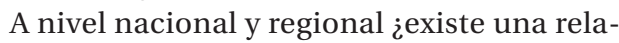
ción negativa entre la situación alimentaria y la poblacional? La situación educacional, sanitaria, de empleo, ambiental $¿$ avalan la necesidad de una política explícita de población? El gobierno sabe y puede ¿cuándo, cómo, bajo qué criterios aplicar una política de población? ¿Existe alternativa válida a la implantación de una política de población?

En realidad, pareciera que en nuestro país no se ha debatido suficientemente el por qué de una política de población. Existe un estado de indefinición tal - conceptual y operativa que tanto las instituciones públicas como las privadas, en la práctica, hacen lo que quieren y más frecuentemente, lo que pueden. Si se corresponde con los programas, está bien, si no, igual está bien, por que de algún siempre es insuficiente. Por lo demás, los organismos oficiales persisten absurdamente en requerir idoneidad ideológica antes que profesional, lo que reproduce la inopia operativa y vuelve a repetirse el ciclo de la inefectividad.

Dados los condicionamientos que se desprenden del planteamiento básico de considerar los problemas de población en su aspecto demográfico, del nivel de conciencia existente, de la viabilidad política y económico-financiera y de la capacidad de gestión de los organismos involucrados ¿qué diferencia habría entre una configuración futura con política y sin política de población?

Por otra parte, los estudios demográficos nacionales ipermiten determinar con exactitud el estado inicial (base line) a partir del cual se puede diseñar un modelo hacia al cual dirigirnos?

Finalmente, de acuerdo a numerosas conclusiones y recomendaciones de organismos nacionales e internacionales, una política de población sólo puede tener éxito cuando se integra a políticas de desarrollo socio-económico exitosas: ¿ cómo sería el caso paraguayo que además de adolecer de planificación económico-social insuficiente sufre enormes conflictos sociales y una acentuación de la pobreza crítica? 


\section{Agradecimiento}

Al Dr. Esteban Ferro, del Comité Científico del Instituto de Investigaciones en Ciencias de la Salud, por la traducción del resumen.

\section{Referencias}

CEPEP/DHS (Centro Paraguayo de Estudios de Población/Demographic and Health Surveys), 1990. Encuesta Nacional de Demografía y Salud. Asunción: Cepep.

CEPEP/CDC/USAID (Centro Paraguayo de Estudios de Población/Centros para el Control de Enfermedades/Agencia de los Estados Unidos para el Desarrollo Internacional), 1996. Paraguay: Encuesta Nacional de Demografía y Salud Reproductiva 1995-1996 (Cepep-Endsr-95/96). Informe Preliminar. Asunción: Cepep.

DGECE (Dirección General de Estadísticas, Censos, y Encuestas), 1950. Censo Nacional de 1950. Asunción: Secretaría de Planificación de la Presidencia de la República.

DGECE (Dirección General de Estadísticas, Censos, y Encuestas), 1950. Censo Nacional de 1962. Asunción: Secretaría de Planificación de la Presidencia de la República.

DGECE (Dirección General de Estadísticas, Censos, y Encuestas), 1950. Censo Nacional de 1972. Asunción: Secretaría de Planificación de la Presidencia de la República.

DGECE (Dirección General de Estadísticas, Censos, y Encuestas), 1950. Censo Nacional de 1982. Asunción: Secretaría de Planificación de la Presidencia de la República.

DGECE (Dirección General de Estadísticas, Censos, y Encuestas), 1950. Censo Nacional de 1992. Asunción: Secretaría de Planificación de la Presidencia de la República.

DGEC (Dirección General de Estadística y Censos), 1981. Encuesta Nacional de Fecundidad, 79, Asunción: Secretaría de Planificación de la Presidencia de la República.
MSPBS/OPS (Ministerio de Salud Pública y Bienestar Social/Oficina Panamericana de la Salud), 1989. Tasas de Mortalidad Materno-Infantil. Asunción: MSPBS. (mimeo.)

MSPBS/ONU/FNUAP/OPS (Ministerio de Salud Pública y Bienestar Social/Naciones Unidas, Fondo de Población/Organización Panamericana de la Salud), 1996. Plan Nacional de Salud Reproductiva del Gobierno del Paraguay. Asunción: MSPBS.

ONU (Organización de las Naciones Unidas), 1974. Población y Desarrollo en América Latina. Ginebra: Consejo Económico y Social, Comisión para América Latina.

ONU/CEPAL/CELADE (Organización de las Naciones Unidas), 1993. Población Equidad y Transformación Productiva. Santiago de Chile: ONU.

SAS (Secretaría de Acción Social), 1996. Plano de Desarrollo Social del Paraguay: una Convocatoria a la Sociedad Paraguaya. Asunción: Presidencia de la República.

STP (Secretaría Técnica de Planificación), 1976. Plan Nacional de Desarrollo Económico y Social 19771981. Síntesis del Diagnóstico Socio-económico. Tomo I. Diciembre. Asunción: Presidencia de la República.

STP (Secretaría Técnica de Planificación), 1984. Plan nacional de Desarrollo. Período 1985-1989. Síntesis del Diagnóstico Global y Sectorial. Tomo I. Versión Preliminar. Octubre. Asunción: Presidencia de la República.

VELAZQUEZ, R. E., 1972. La Población del Paraguay en 1962. Asunción:Centro Paraguayo de Estudios Sociológicos. Colección de Reimpresiones. 